\title{
Disk Structures from Triaxial Tumbling Halos
}

\author{
Dalia Chakrabarty ${ }^{1}$ and John Dubinski ${ }^{2}$ \\ ${ }^{1}$ School of Physics \& Astronomy, University of Nottingham, U.K., \\ email: dalia.chakrabarty@nottingham.ac.uk, \\ ${ }^{2}$ Dept. of Astronomy \& Astrophysics, University of Toronto, CITA, Canada
}

\section{Motivation \& Methodology}

In this work, we investigate the dynamical influence that external, triaxial and tumbling halos may exercise on disks that may lie embedded in them. Such halo configurations are noted in cosmological simulations, to typically rotate about their short axes, with tumbling periods of the order of a Hubble time. Thus, such halos can exert a slowly changing external torque on disks, affecting disk evolution in the process. Another source of complex dynamical effects is the probable misalignment between a disk and a triaxial dark halo. In this work, we conduct a series of experiments in which we explore the effect of a slowly changing quadrupolar tidal potential on N-body models of our Galaxy and M31; our particular aim is to check if this can induce disk structures such as bars, spirals and warps!

We measure the expected strengths of the quadrupolar tidal field of the halo directly from halos within cosmological simulations that are performed in the currently accepted $\Lambda C D M$ paradigm. Our simulations indicate that that the tidal torque on the Milky Way disc from the external halo is an order of magnitude larger than that due to the LMC. We support our numerical findings with analytical calculations.

\section{Results \& Conclusions}

We find that the torques are strong enough to induce warps in disks similar to those observed in real spirals and also induce the bar instability at later times in Galaxy models that are otherwise stable for long periods of time in isolation; we also observe forced spiral structure near the edge of the disk (Figure 1). This overlooked influence of dark halos may well be responsible for many of the peculiar aspects of disk galaxy dynamics. 

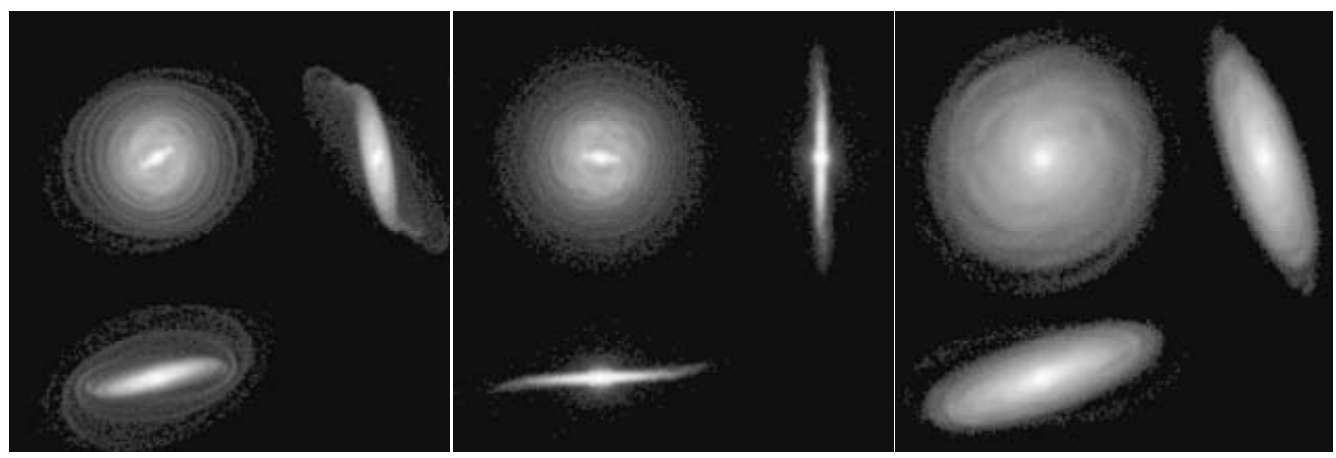

Figure 1. The $x-y$ (top left), $y-z$ (top right) and $x-z$ (bottom left) views of the Milky Way (left and middle panels) and the M31 disk (right) at nearly the end (time $66 \mathrm{Gyr}$ ) of our N-body simulations, performed with a halo that is misaligned with the disk by an angle of $30^{\circ}$. The left and right panels correspond to a halo pattern speed of about $0.12 \mathrm{kms}^{-1} \mathrm{kpc}^{-1}$ while the tumbling rate for the middle panel is 3 times higher. The middle panel is also characterised by a quadrupolar strength that is quarter of that used in the runs corresponding to the other two. A general result is that at a given time, the faster the halo tumbles, the higher is the strength of the bar that is induced in the Milky Way disc. The M31 disk does not form a bar on being torqued this way; also a strong warp sets in early and is visible till about 5Gyrs in M31 unlike in the Milky Way model, in which the warp is sustained till the end of our simulation. Flocculent spiral arms are noticed till the edge of the disk, where normally, self-gravity is too weak to bear this structure. 